\title{
Treatment of Colorectal Cancer with Natural Anal Passage-based Fast Track Surgery
}

\author{
SHIZHU HAO*
}

Department of Oncology Surgery, Tangshan Gongren Hospital, No. 27 Wenhua Road, Lubei District, Tangshan City, 063000, Hebei Province, China

\section{Hao: Colorectal Cancer and Fast Track Surgery}

\begin{abstract}
In this study, 200 patients with colorectal cancer surgery from January 2013 to June 2018 were recruited and divided into the rapid rehabilitation treatment group and the control group. Basic information was collected and albumin expression level is analysed. In addition, the pain grading method was used to grade the postoperative conditions to observe the treatment effect of the rapid rehabilitation treatment group and control group. The results showed that there is no statistical difference between the two groups in the basic situation and previous history of the two groups. In the analysis of albumin expression level, significant statistical differences were found between the two groups and at different time points. And in the analysis of postoperative pain levels of patients in the two groups, it was found that the proportion of taking painkillers in the rapid rehabilitation treatment group is significantly lower, and with the increase of postoperative time, the final pain levels of patients in the two groups show a statistical difference $(p<0.05)$. Therefore, it is found that rapid rehabilitation is indeed better than the traditional perioperative treatment in this study. Although there are some deficiencies in the experimental process, it still provides a new experimental reference basis for the treatment of colorectal cancer in the future.
\end{abstract}

Key words: Rapid rehabilitation therapy, colorectum, albumin, pain grading

Colorectal cancer is the third most common cancer in the world ${ }^{[1]}$. With acceleration of industrialization, air pollution is becoming more and more severe and people are paying more and more attention to health. Colorectal cancer is a relatively common clinical malignant tumor of the digestive system in China, which poses a serious threat to human health and is one of the five most common malignant tumors in $\mathrm{China}^{[2,3]}$. In the perioperative period of traditional colorectal cancer patients, many measures or in practice without the support of relevant scientific evidence-based medicine ${ }^{[4]}$. With recent developments in medical technology, the level of medical care is also improving. In order to reduce the incidence of various complications in the perioperative period and the recovery time of patients, the fast track surgery (FTS) concept gradually introduced by surgeons ${ }^{[5]}$.

Colorectal cancer often can't be detected due to the absence of obvious and specific clinical symptoms in the early stage, so early detection, early diagnosis and early treatment are of great significance for improving prognosis and reducing mortality ${ }^{[6]}$. Investigations and studies have shown that patients with FTS implemented according to clinical guidelines feel significantly better in pain and discomfort and the length of hospital stay is significantly shortened, which has no obvious adverse effect on patients' quality of life and comfort $^{[7,8]}$. However, some scholars believe that there is a big difference between the concept of FTS and the traditional perioperative rehabilitation treatment of colorectal cancer and some of the FTS measures are even considered to be against the conventional practice ${ }^{[9]}$. Other studies claim that there is no significant difference between the clinical effects of FTS and the standard regimen, similar clinical results can also be obtained by not fully following the measures recommended by FTS, and the traditional regimen is simpler and easier to operate ${ }^{[10]}$. With the maturity of laparoscopic minimally invasive technology and the embodiment of the advantages of laparoscopic radical surgery for colorectal cancer, the combined application of FTS in the treatment of colorectal cancer patients has been promoted to a greater extent. Controversy and research on this joint program are still underway to 
further explore the value of FTS in laparoscopic radical resection of colorectal cancer.

To sum up, in order to study the treatment of colorectal cancer via natural anal passage based on FTS, patients undergoing colorectal cancer surgery from January 2013 to June 2018 are selected. The patients are divided into groups and relevant information is collected for statistical analysis to observe the therapeutic effect of the rapid rehabilitation treatment group and the control group, so as to provide a reference direction for the treatment of colorectal cancer in the future.

In this study, 200 patients with colorectal cancer admitted to the gastrointestinal surgery department of Tangshan Gongren Hospital from January 2013 to June 2018 were selected. According to different perioperative treatment measures, the patients are divided into the rapid rehabilitation surgery and the control group for traditional intervention measures. Among them, 115 patients were in the fast recovery surgery group and 85 patients in the traditional treatment group. Informed consent was signed by the patients or their family members was obtained and this study was approved by the medical ethics committee of Tangshan Gongren Hospital.

Inclusion criteria included patients aged 40-70 y; patients confirmed by colonoscopy, biopsy and pathology, and no lymph nodes and distant organs and organs metastasis are found by chest radiography, abdominal ultrasound, abdominal CT and other examinations; patients who have not received adjuvant treatment before surgery and are willing to cooperate with the study and sign informed consent.

Exclusion criteria were patients who have been on diet restriction, indwelling gastrointestinal decompression tube and nutritional therapy before admission; patients undergoing emergency surgery for intestinal obstruction or bleeding or failing laparoscopic surgery; patients with cardiorespiratory and renal disease and poor systemic nutrition; patients with a history of cardiac and abdominal surgery; patients who have received preoperative chemoradiotherapy; patients who underwent multiple protein or blood transfusions preoperatively, intraoperatively, and postoperatively; patients who need to remove other organs at the same time; patient who has not been cured; patients who failed to perform a one-stage anastomosis; patient who has suffered from mental illness; patients who have been transferred, planted or disseminated in the distance; and patients with incomplete data.
Demographic data included gender, age, height, weight, ethnicity, education level, occupation, economic status, personality type and religious belief. Data related to the disease included previous hospitalization frequency and disease name, previous surgery history, chronic pain history, drug history, drinking history, and preoperative diagnosis of this hospitalization.

According to different perioperative treatment measures, the patients were divided into the rapid rehabilitation surgery group and the control group for traditional intervention measures. The fast rehabilitation surgery group underwent laparoscopic minimally invasive surgery to give optimized preoperative intestinal preparation, optimized intraoperative anesthesia and analgesia program, and did not have routine gastrointestinal decompression or abdominal drainage tubes. After the operation, the rehabilitation was strengthened for these patients and the patients could be discharged if their movement out of bed and feeding behavior reached discharge standard. The control group underwent laparoscopic minimally invasive surgery but had traditional treatment interventions.

First of all patients in the rapid rehabilitation surgery group were provided with health education regarding the causes, development and prognosis of the tumor and explaining the postoperative measures to promote rehabilitation as well as the time needed for each stage of rehabilitation. Intestinal preparation was performed. Polyethylene glycol electrolyte powder was given orally $12 \mathrm{~h}$ before surgery, routine mechanical intestinal preparation was not performed, the slag-free diet was provided one day before surgery and $10 \%$ glucose was given orally $3 \mathrm{~h}$ before surgery. Gastrointestinal decompression was not performed during the treatment and general anesthesia combined with continuous epidural anesthesia was given under endotracheal intubation. During the laparoscopic surgery heat preservation and infusion volume was controlled. Drainage tube was not placed and liquid diet was also provided on the next day of surgery.

For the control group of patients, health education was provided about the etiology, development and prognosis of the tumor, but the routine surgical treatment is introduced. Intestinal preparation was performed, oral antibiotics were given before surgery, mannitol and sugar saline were given one day before the operation, enema of soap and water was given in the morning on the day before surgery, fluid diet is provided $3 \mathrm{~d}$ before and the night before surgery diet and water was prohibited. During the course of treatment, routine 
gastrointestinal decompression was performed and general anesthesia was performed under endotracheal intubation. Laparoscopic surgery was performed without heat preservation and fluid volume control. Drainage tube was placed routinely and the diet was given after intestinal exhaust.

The degree of pain in patients of both groups at 24, 48 and $72 \mathrm{~h}$ after surgery was observed with the pain digital score quadruple numerical rating score 10 , with 0 indicating no pain and 10 indicating unbearable pain. Assessment grade was as follows, mild pain (1 to 3 points); moderate pain (4 to 6 points); severe pain (7 to 10 points). The observation indices included the time of first anal exhaust and defecation, time of getting out of bed, hospitalization time, postoperative medication, postoperative drainage tube use, hospitalization cost and complications and blood biochemical indices included albumin before and 1,2 and $4 \mathrm{~d}$ after surgery.

The data of this study were analyzed using SPSS 21.0 statistical analysis software. Variables that conform to the normal distribution are expressed as mean \pm standard deviation (SD), and comparison between groups is performed by $t$ test or repeated measures of variance analysis; the variables that don't conform to the normal distribution are expressed by the median and quartile (M, IQR), and the rank sum test is used for the comparison between groups. Counting data are compared by chi-square test or Fisher exact probability test. All the statistical tests are bilateral, and the test level is $\alpha=0.05$.
A total of 200 patients with colorectal cancer who were admitted to the gastrointestinal surgery department of Tangshan Gongren Hospital were investigated in this study. There were 115 patients in the rapid rehabilitation surgery group, including 69 male and 46 female patients, and 85 patients in the control group, including 49 male and 36 female patients. The average age of patients in the rapid rehabilitation surgery group was 58.7 $\pm 6.52 \mathrm{y}$, with 59 cases of colonic laparoscopic surgery and 56 cases of rectal laparoscopic surgery. The average age of the patients in the control group was 58.4 $\pm 7.57 \mathrm{y}$, including 38 cases of colonic laparoscopic surgery and 47 cases of rectal laparoscopic surgery. There is no statistical difference in each baseline feature between the fast-rehabilitation surgery group and the control group, as shown in Table 1.

The preoperative history of the two groups was compared indicating 43 patients with hypertension, 35 with diabetes and 24 with cardiovascular disease in the rapid rehabilitation surgery group. In the control group, there were 31 patients with hypertension, 23 with diabetes and 12 with cardiovascular disease. There was no statistical difference between the two groups in terms of previous medical histories, as shown in Table 1.

Table 2 shows data related to postoperative hospitalization time, postoperative drinking water time, postoperative diet time, postoperative first exhaust time, postoperative first defecation time and postoperative activity time of getting out of bed were all statistically

TABLE 1: COMPARISON OF BASIC INFORMATION OF PATIENTS

\begin{tabular}{|c|c|c|c|c|c|}
\hline Item & & ERAS $(n=115)$ & Control group $(n=85)$ & $t / x^{2}$ & $P$ value \\
\hline Gender (male/female) & & $69 / 46$ & $49 / 36$ & 0.004 & 0.931 \\
\hline Age & & $58.7 \pm 6.52$ & $58.4 \pm 7.57$ & 0.117 & 0.925 \\
\hline \multirow{2}{*}{ Surgical method } & Colon & 59 & 38 & \multirow{2}{*}{1.472} & \multirow{2}{*}{0.223} \\
\hline & Rectum & 56 & 47 & & \\
\hline \multirow{3}{*}{ Past medical history [n] (\%) } & Hypertension & $43(37.4)$ & $31(36.5)$ & 0.251 & 0.634 \\
\hline & Diabetes & $35(30.4)$ & $23(27.1)$ & 0.072 & 0.795 \\
\hline & $\begin{array}{c}\text { Cardiovascular } \\
\text { diseases }\end{array}$ & $24(20.9)$ & $12(14.1)$ & 1.583 & 0.207 \\
\hline
\end{tabular}

TABLE 2: COMPARISON OF POSTOPERATIVE RECOVERY OF PATIENTS

\begin{tabular}{|c|c|c|c|c|}
\hline Item & ERAS $(n=115)$ & Control group $(n=85)$ & $t / Z^{*}$ & $P$ value \\
\hline Postoperative hospitalization time (d) & $7.3 \pm 2.1$ & $8.6 \pm 3.9$ & -3.145 & 0.002 \\
\hline Postoperative drinking time (d) & $1.7 \pm 0.9$ & $2.2 \pm 0.7$ & -5.417 & $<0.001$ \\
\hline Postoperative diet time $(\mathrm{d})$ & $1.9 \pm 0.6$ & $2.5 \pm 0.8$ & -7.895 & $<0.001$ \\
\hline First exhaust time $(\mathrm{h})$ & $38(24,49)$ & $39(35,61)$ & -2.069 & $<0.001$ \\
\hline First defecation time $(\mathrm{h})$ & $44(26,58)$ & $46.2(31,76)$ & -4.852 & 0.0027 \\
\hline Time to start getting out of bed (d) & $1.7 \pm 0.64$ & $2.3 \pm 0.59$ & -7.247 & $<0.001$ \\
\hline
\end{tabular}


significant $(\mathrm{p}<0.05)$. Among them, the postoperative hospitalization time was $7.3 \pm 2.1 \mathrm{~d}$, the postoperative drinking time was $1.7 \pm 0.9 \mathrm{~d}$, the postoperative diet time was $1.9 \pm 0.6 \mathrm{~d}$, and the first postoperative exhaust median time is $38 \mathrm{~h}$, the median time of first defecation was $44 \mathrm{~h}$, and the time to get out of bed after operation was $1.7 \pm 0.6 \mathrm{~d}$ in the rapid rehabilitation surgery group and the postoperative hospitalization time was $8.6 \pm 3.9 \mathrm{~d}$, the postoperative drinking time was $2.2 \pm 0.7 \mathrm{~d}$, the postoperative diet time was $2.5 \pm 0.8 \mathrm{~d}$, and

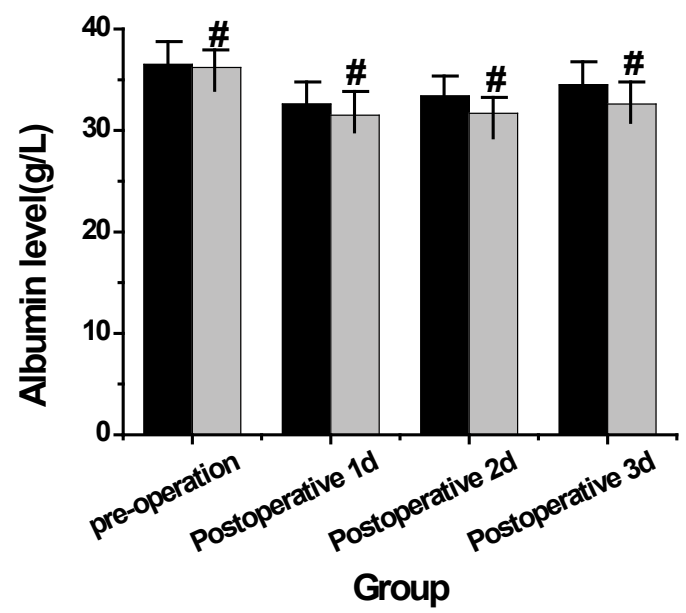

Fig. 1: Albumin levels in both groups of patients Albumin levels in the rapid rehabilitation surgery group and the control group (\#p<0.05 compared to the control group); ( $\square$ ) ERAS group, ( $($ ) control group
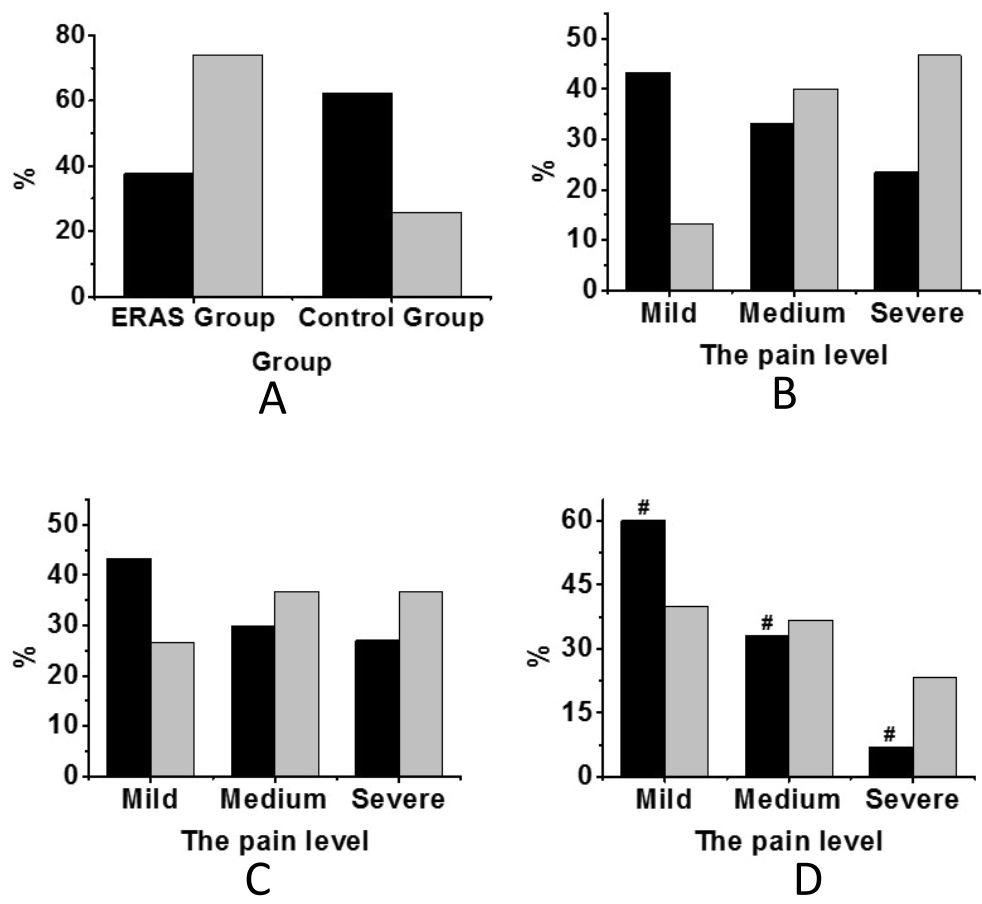

Fig. 2: Postoperative pain in both groups of patients

Histogram of postoperative pain the rapid rehabilitation surgery group and the control group. A. use of painkillers, B. comparison of degree of pain on day 1 after surgery, C. comparison of degree of pain on day 2 after surgery, D. comparison of degree of pain on day 4 after surgery. \#P<0.05 compared to the control group. A: (घ) Pain killers used, ( $\square$ ) not used; B, C, D: (घ) ERAS group, ( $\square$ ) control group 
of patients in the 2 groups, it can be concluded that the pain situation of the fast recovery surgery group is significantly better than that of the control group, which indicates that the fast recovery surgery group has a better recovery ability.

To study the effect of colorectal cancer treatment by natural anal passage-based FTS, patients with colorectal cancer admitted to the gastrointestinal surgery department of Tangshan Gongren Hospital from January 2013 to June 2018 were selected. The basic information of these subjects is collected, albumin levels were analyzed and postoperative conditions were graded with the pain grading method so as to observe the therapeutic outcome of the rapid rehabilitation treatment group and the control group. Results showed that there is no statistical difference between the 2 groups in the analysis of the basic situation and previous history of the 2 groups. In the analysis of albumin expression level, there were significant statistical differences between the 2 groups at different time points. In the analysis of postoperative pain levels of patients in the two groups, it was found that the proportion of patients taking painkillers in the rapid rehabilitation treatment group was significantly lower and with the increase of postoperative time, the final pain levels of patients in the two groups showed a statistical difference $(p<0.05)$.

In summary, study on the treatment of colorectal cancer via natural anal passage-based FTS, it was found that the rapid rehabilitation treatment was indeed superior to the traditional perioperative treatment, which provided a new experimental reference basis for the treatment of colorectal cancer. However, there were some deficiencies in the research study, such as insufficient sample size. Going forward, the sample size should be increased to further study the clinical application of rapid rehabilitation therapy.

\section{REFERENCES}

1. Walkiewicz K, Nowakowska-Zajdel E, Strzelczyk J,
Dzięgielewska-Gęsiak S, Muc-Wierzgoń MS. levels of ADAM10, ADAM12, ADAM17 AND ADAM28 in colorectal cancer patients. J Biol Regul Homeost Agents 2017;31(4):92934.

2. Rong LU, Junlei LI, Jia P, Effects of Rehabilitation Surgery Assisted to FOLFOX Chemotherapy on Postoperative Recovery, Complica-tions and Quality of Life in Patients with Colorectal Cancer. China Pharm 2016;27(27):3774-76.

3. Qiu Y, Luo Y, Qin S, A Randomized Controlled Study on Efficacy of Perioperative Administration of Houpupaiqi Mixture on Fast Track Surgery in Patients with Laparoscopic Colorectal Cancer. Chinese J Gastroenterol 2018; 23(3):14851 .

4. Ding C, Peng J, Qiu J, Clinical analysis of total laparoscopic resection for colorectal cancer by NOSES: a report of 50 cases. China J Endoscopy 2018; 24(6): 97-101.

5. Liu Y. Application of fast track surgery in routine nursing for patient with colorectal cancer. Saudi J Biol Sci 2017;24(8):1939-1942.

6. Lin KY, Denehy L, Granger CL, Pelvic floor outcomes in patients who have undergone general rehabilitation following surgery for colorectal cancer: A pilot study. Physiother Theory Pract 2019;35(3):206-218.

7. Deng H, Liu D, Mao X, Phosphodiesterase-5 inhibitors and vacuum erection device for penile rehabilitation after laparoscopic nerve-preserving radical proctectomy for rectal cancer: A prospective controlled trial. Am J Mens Health 2017;11(3):641-46.

8. Wang XZ, Lu LH, Deng HY. Effect of nursing intervention on early rehabilitation of patients with abdominal surgery. J. Hainan Med. Univ 2016;22(11):150-51.

9. Pereira J, Paduraru M. Multimodal Rehabilitation in Geriatric Emergency Surgery. Chirurgia (Bucharest, Romania: 1990), 2017;112(5):558-565.

10. Abbasi J. Rapid test for antibiotic susceptibility. JAMA 2017;318(14):1314-14.

This is an open access article distributed under the terms of the Creative Commons Attribution-NonCommercial-ShareAlike 3.0 License, which allows others to remix, tweak, and build upon the work non-commercially, as long as the author is credited and the new creations are licensed under the identical terms

This article was originally published in Special issue on "Trends in therapeutic Management of Various Conditions" Indian J Pharm Sci 2020:82(3) spl issue 6;77-81 